\title{
Food avoidance in an Australian adult population sample: the case of dairy products
}

\author{
Bella Yantcheva ${ }^{1,2}$, Sinéad Golley², David Topping ${ }^{2}$ and Philip Mohr ${ }^{1, *}$ \\ ${ }^{1}$ School of Psychology, University of Adelaide, Adelaide, SA 5005, Australia: ${ }^{2}$ CSIRO Food and Nutrition Flagship, \\ Adelaide BC, South Australia, Australia
}

Submitted 14 August 2015: Final revision received 6 October 2015: Accepted 19 0ctober 2015: First published online 20 November 2015

\begin{abstract}
Objective: To assess the prevalence of and explanations for the avoidance of dairy foods, including symptoms attributed to their consumption, diagnoses and psychological predictors of avoidance. Also considered were comparisons with symptom-related avoidance of wheat in the same sample.

Design: Cross-sectional population survey.

Setting: The study was conducted in Australia using a national postal omnibus survey.

Subjects: Adults aged 18 years and over ( $n$ 1184; 52.9\% female) selected at random from the Australian Electoral Roll.

Results: Despite few claims of formally diagnosed allergy or intolerance, $11.8 \%$ of the sample reported avoiding dairy products because of adverse physiological effects, which commonly included gastrointestinal symptoms. Unlike wheat (3.5\%) or wheat-and-dairy (3.6\%) avoidance, dairy avoidance (8.2\%) was predicted by age (negatively) and worry about illness (positively).

Conclusions: The findings are further evidence of a widespread tendency for consumers to exercise control over their health by eliminating dietary factors considered suspect without medical evidence or oversight. Unanswered questions include the decision processes underlying dairy avoidance, whether symptoms are attributed correctly, the agents and physiological mechanism(s) involved, the relative contributions of symptom severity and vigilance to the association with illness worry, and the nutritional adequacy of dairy avoiders' diets. Irrespective of the accuracy of self-diagnoses, if the elimination of suspect foods is an end in itself the paradoxical possibility for nutritional imbalances may have significant public health implications.
\end{abstract}

\section{Keywords Dairy avoidance Lactose intolerance Dairy allergy Health decision making}

The benefits of the consumption of dairy foods are numerous. They are the biggest source of $\mathrm{Ca}$ in the Australian diet and whole milk and other dairy foods provide a unique package of other essential nutrients including protein, vitamins (A, $\mathrm{B}_{12}$ and riboflavin) and minerals ( $\mathrm{P}, \mathrm{Mg}, \mathrm{K}$ and $\mathrm{Zn})^{(1)}$. Not consuming these foods risks insufficient dietary $\mathrm{Ca}$ intake and has been known to result in reduced bone mineral density, increased incidence of fracture, and other risks to health and well-being ${ }^{(1-3)}$.

National information about the prevalence and drivers of avoidance of dairy products is scarce in Australia. The Australian Bureau of Statistics reported that in 2011-12, $17 \%$ of Australians aged 2 years or over ( 3.7 million people) reported avoiding a food due to allergy or intolerance, with cow's milk and other dairy products ( $4.5 \%$ ) being the most commonly claimed source of intolerance ${ }^{(4)}$. Because these figures relate only to cases of food avoidance specifically attributed by interviewees to an allergy or intolerance, they can be expected to underestimate - perhaps substantially the population prevalence of avoidance of any given food, including dairy foods.

In a national population survey, $7 \%$ of apparently noncoeliac Australian adults reported avoiding consumption of products containing wheat for the alleviation of physical symptoms. In the great majority of cases, these related to the gastrointestinal system and included those that are diagnostic of irritable bowel syndrome (IBS) according to the ROME 2 criteria. Wheat avoidance occurred overwhelmingly in the absence of a formal diagnosis and was predicted by receptiveness to complementary medicine (positively) and conventional medicine (negatively) but not by measures of anxiety or illogical reasoning style. Questions arising from these findings relate, inter alia, to the possible physiological mechanisms underlying symptoms, the decision processes leading to self-prescribed avoidance of wheat and the potential for nutritional imbalances ${ }^{(5)}$. 
Compounding these questions, however, is the additional finding that $53 \%$ of self-identified symptomatic wheat avoiders also reported, in a separate sub-section of the same omnibus survey, that they were avoiding consuming dairy foods. This points to a possibly widespread phenomenon of poly-avoidance with significant implications for both the development of nutritional imbalances through the compensatory overconsumption of other foods as well as the avoidance - and the task of identifying a specific food component as a source of symptoms. Where wheat avoidance and dairy avoidance are concerned, it also prompts the question whether these are discrete or intrinsically related phenomena.

Using data from the same survey, the present paper addresses the prevalence of and explanations for dairy avoidance, including reported symptoms, diagnoses and influences, as well as possible psychological predictors of and health conditions correlated with dairy avoidance behaviour. This is the same set of measures as used in the wheat avoidance study ${ }^{(5)}$. The psychological characteristics considered thus include neuroticism, tendency to worry about illness, propensity for a less analytical and/or more intuitive reasoning style, and receptiveness to each of conventional and complementary medicine. Of key interest throughout are commonalities with and differences from the case of wheat avoidance.

\section{Method}

\section{Participants and study design}

Data for the current study were obtained from a crosssectional survey of attitudes and behaviours related to health, diet, foods and food characteristics in an adult Australian population sample selected at random from the Australian Electoral Roll. The data were collected through the Commonwealth Scientific and Industrial Research Organisation's (CSIRO) 2010-11 Food and Health Survey. Detailed data collection methods and procedure are described elsewhere ${ }^{(5)}$. One thousand one hundred and eighty-four of a possible 2867 postal surveys were returned completed (an overall response rate of $41.3 \%$ ). The sample consisted of adults aged 18 years and over: 527 males (44.5\%), 626 females (52.9\%) and thirty-one not specified (2.6\%); mean reported age was 51.64 (SD 16.78) years. Women $\left(\chi^{2}(1, n\right.$ 1148) $=6 \cdot 02, P=0 \cdot 01)$ and older people $\left(\chi^{2}(6, n 1144)=149 \cdot 78, P<0 \cdot 01\right)$ were over-represented relative to the population; the data were weighted to adjust for this in the calculation of population prevalence estimates.

\section{Procedure}

The CSIRO Food and Health Survey was a 200-item postal omnibus survey distributed nationally in December 2010; participation was anonymous. The study was conducted according to the guidelines laid down by the Declaration of Helsinki and the research protocol was approved by the CSIRO Human Research Ethics Committee.

\section{Variables}

The questionnaire contained a section labelled 'Avoidance of wheat and/or dairy products'. Responses to the avoidance of wheat products are reported elsewhere ${ }^{(5)}$; an identical set of questions addressing the avoidance of dairy products is the focus of the present paper. Questions asked whether the participant avoided all or certain dairy products and to specify which; to state the main reason for avoiding the consumption; to identify any reactions to dairy consumption from a list of eighteen physiological and psychological symptoms; to indicate on a 3-point scale ('not at all', 'somewhat', 'very much') the extent to which each of seven medical or non-medical sources of recommendation or information had contributed to their decision to avoid dairy; and to identify any formally diagnosed condition that required them to avoid dairy.

Other sections of the survey provided the following measures. Neuroticism, a personality dimension characterised by anxiety and negative emotions, was measured with nine items from the NEO-N Domain ${ }^{(6)}$. Participants rated statements (e.g. 'I panic easily') on a 5-point scale, where $1=$ 'completely false' and $5=$ 'completely true'. Reasoning style was assessed by the Rational-Experiential Inventory $(\mathrm{REI})^{(7)}$, comprising five items drawn from each of the Need for Cognition ${ }^{(8)}$ and Faith in Intuition Scales ${ }^{(7)}$, each item employing a 5-point scale from 'completely false' to 'completely true'. Higher scores represent increased reliance on analytical and intuitive reasoning for each scale, respectively.

In a section addressing general health, participants were asked to identify past diagnoses from a list of twelve conditions. Illness worry was measured with four items from the Whitely Index for the assessment of hypochondriasis (e.g. 'Do you often worry about the possibility that you have got a serious illness?') ${ }^{(9)}$. Receptiveness to conventional medicine (four items) and complementary medicine (six items) were assessed by ratings of the likelihood of taking certain actions for health matters (e.g. 'Consult a doctor', 'Consult a naturopath'). Responses were measured on a scale from $1=$ 'definitely not' to $7=$ 'definitely'.

\section{Statistical analyses}

Prior to analyses, the pattern of missing data for the continuous variables was investigated with the Missing Values Analysis module in the statistical software package IBM SPSS Statistics version 20.0. Little's MCAR test ${ }^{(10)}$ showed that the data were missing completely at random $\left(\chi^{2}=28701 \cdot 40, \mathrm{df}=28818, P=0 \cdot 69\right)$; consequently, missing values were imputed using the EM (expectation maximization) algorithm. Descriptive statistics were calculated to provide frequency distributions and 
cross-tabulations of key variables. The $\chi^{2}$ test of independence and Fisher's exact test, as appropriate ${ }^{(11)}$, were used to examine associations between categorical variables. Predictors of avoidance were assessed by means of multivariable logistic regression. Pearson point-biserial correlations were calculated for bivariate associations between continuous and dichotomous variables, and group differences in ratings of sources of influence were assessed with one-way ANOVA. IBM SPSS Statistics versions $20 \cdot 0-22 \cdot 0$ were used for all analyses.

\section{Results}

\section{Preliminary analyses}

Of the 1184 participants who returned the completed questionnaires, 188 (unweighted 15.9\%, weighted 16.6\% of the sample) indicated that they were currently avoiding dairy products (seventy men, 115 women and three of undisclosed gender). Of this number, fifteen (1.3\% unweighted, $1.2 \%$ weighted) reported avoiding dairy products because of a medically diagnosed condition, mostly cardiovascular, and thirty-four (2.9\% unweighted, $2 \cdot 8 \%$ weighted) gave diverse reasons unrelated to symptoms or diagnosed conditions. Explanations given included: concerns about levels of saturated fat; personal dislike of taste; calorie and weight management; and the view that dairy foods were not appropriate for human adult consumption.

The remaining 139 (11.7\% unweighted, 12.6\% weighted) reported symptoms and negative reactions to the consumption of dairy products, including nine people $(0.8 \%)$ who reported having been formally diagnosed with coeliac disease. All subsequent analyses focused on the remaining 130 (11.0\% unweighted, $11.8 \%$ weighted) symptomatic dairy avoiders: eighty-two (63.1\%) women, forty-five $(34.6 \%)$ men and three $(2 \cdot 3 \%)$ of unknown gender.

\section{Main analyses}

Of this symptomatic dairy-avoiding group, seventy-eight (60.0\%) nominated physical reactions as their main reason for avoiding dairy foods, twenty-five (19.2\%) nominated intolerance or allergy, five (3.9\%) gave various other reasons and twenty-two (16.9\%) gave no reason. Partial dairy avoidance ( $n$ 98; 75.4\%) was reported more frequently than full avoidance ( $n 29 ; 22.3 \%$ ). The foods most commonly nominated by partial avoiders were milk (55.4\%), cheese (16.9\%) and cream (16.2\%).

Thirty (23.1\%) symptomatic dairy avoiders reported having a formal diagnosis that required them to avoid dairy. The diagnoses were reported as lactose intolerance ( $n$ 5; 3.8\%), high cholesterol ( $n$ 6; 4.6\%), asthma ( $n$; $3 \cdot 1 \%$ ), allergy ( $n 3 ; 2 \cdot 3 \%$ ), IBS ( $n 3 ; 2.3 \%$ ), diabetes ( $n$ 2; $1.5 \%)$ and several unique responses.

\section{Symptoms reported}

The most commonly reported reactions involved gastrointestinal discomfort, with $62.3 \%$ indicating either 'stomach discomfort or cramps' or 'bloating or wind'; also common were diarrhoea and mucus build-up. Table 1 lists the frequency of occurrence of all eighteen symptoms; these did not differ significantly between self-reported complete and partial avoiders.

The cross-tabulation of dairy avoidance with wheat avoidance data identified forty-five symptomatic wheat avoiders (SWA; 3.8\% unweighted, 3.5\% weighted), eighty-eight symptomatic dairy avoiders (SDA; $7.4 \%$ unweighted, $8.2 \%$ weighted) and forty-two symptomatic wheat-and-dairy avoiders (SWDA; 3.6\%), the latter representing $32 \cdot 3 \%$ of the 130 symptomatic dairy avoiders. Figure 1 summarises the classification of participants. Table 2 reports a comparison of categories of symptoms attributed to wheat and dairy by avoiders of either, but not both. Relative to wheat, dairy was less frequently associated with gastrointestinal symptoms $\left(\chi^{2}=5.94, P=0.015\right)$ and tiredness $\left(\chi^{2}=7.68, P=0.006\right)$ and more frequently associated with mucus production $\left(\chi^{2}=13.61, P<0.001\right)$. Further comparisons showed that wheat-and-dairy avoiders were more likely than wheat avoiders to attribute mucus production $\left(23.8 \% ; \chi^{2}=9 \cdot 17, P=0.007\right)$ to wheat and more likely than dairy avoiders to attribute tiredness (31.0\%; $\left.\chi^{2}=8.69, P=0.007\right)$ and skin problems or hives $\left(23.8 \% ; \chi^{2}=9 \cdot 15, P=0.004\right)$ to dairy. There were no other group differences.

Table 3 shows the results of multivariable logistic regression for the prediction of membership of the three avoidance categories relative to the rest of the sample ( $n$ 994), coeliac cases excluded. Symptomatic wheat

Table 1 Frequency and type of symptoms reported by symptomatic dairy avoiders ( $n$ 130); CSIRO Food and Health Survey, Australia, December 2010-February 2011

\begin{tabular}{lcc}
\hline & \multicolumn{2}{c}{ Total } \\
\cline { 2 - 3 } Reported symptoms & $n$ & $\%$ \\
\hline Stomach discomfort or cramps & 66 & $50 \cdot 8$ \\
Bloating or wind & 57 & $43 \cdot 8$ \\
Diarrhoea & 48 & $36 \cdot 9$ \\
Mucus build-up & 46 & $35 \cdot 4$ \\
Feeling sluggish or tired & 22 & $16 \cdot 9$ \\
Vomiting or nausea & 21 & $16 \cdot 2$ \\
Constipation & 19 & $14 \cdot 6$ \\
Heartburn or indigestion & 18 & $13 \cdot 8$ \\
Skin problems & 15 & 11.5 \\
Sleep disturbance & 13 & 10.0 \\
Headaches & 13 & 10.0 \\
Breathing problems & 7 & 5.4 \\
Body aches and pains & 7 & 5.4 \\
Feeling anxious or irritable & 4 & 3.1 \\
Sweating & 4 & 3.1 \\
Hives & 4 & $3 \cdot 1$ \\
Feeling restless or hyperactive & 1 & $0 \cdot 8$ \\
Feeling sad or blue & 0 & 0.0 \\
\hline
\end{tabular}

Multiple responses were allowed. 


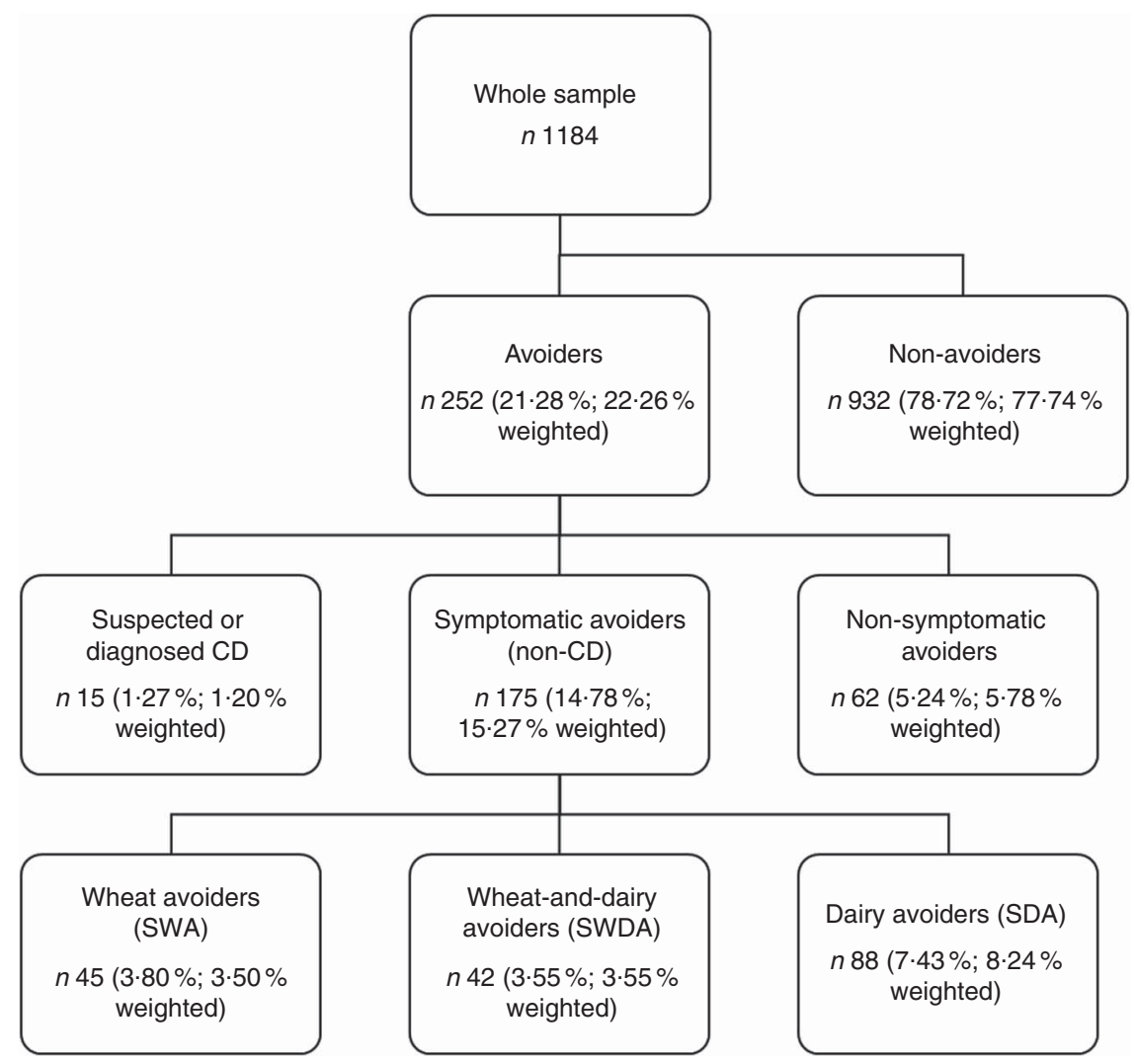

Fig. 1 Flowchart of the classification of self-reported avoiders of dairy products and wheat products; CSIRO Food and Health Survey, Australia, December 2010-February 2011 (CD, coelic disease)

Table 2 Summary of symptoms reported by avoiders of wheat $(n 45)$ and dairy ( $n$ 88); CSIRO Food and Health Survey, Australia, December 2010-February 2011

\begin{tabular}{|c|c|c|c|c|}
\hline \multirow[b]{2}{*}{ Reported symptoms } & \multicolumn{2}{|c|}{ Wheat avoiders } & \multicolumn{2}{|c|}{ Dairy avoiders } \\
\hline & $n$ & $\%$ & $n$ & $\%$ \\
\hline Gastrointestinal* $^{*}$ & 43 & $95 \cdot 6$ & 68 & $77 \cdot 3$ \\
\hline Nausea and vomiting & 3 & $6 \cdot 7$ & 12 & $13 \cdot 6$ \\
\hline Skin problems or hives & 5 & $11 \cdot 1$ & 5 & $5 \cdot 7$ \\
\hline Breathing problems & 1 & $2 \cdot 2$ & 4 & 4.5 \\
\hline Sweating & 2 & 4.4 & 3 & 3.4 \\
\hline Head or body aches & 6 & $13 \cdot 3$ & 11 & 12.5 \\
\hline Behavioural & 9 & $20 \cdot 0$ & 11 & $12 \cdot 5$ \\
\hline Mucus ${ }^{* *}$ & 1 & $2 \cdot 2$ & 28 & 31.8 \\
\hline Sluggish or tired* & 14 & $31 \cdot 1$ & 9 & $10 \cdot 2$ \\
\hline
\end{tabular}

Gastrointestinal = stomach discomfort or cramps; bloating or wind; diarrhoea; constipation; or heartburn or indigestion.

Behavioural = feeling anxious or irritable; feeling restless or hyperactive; or feeling sad or blue.

Significant difference in frequency of reported symptom by group: ${ }^{*} P<0.05$, ${ }^{* *} P<0.001$.

avoidance was significantly predicted by gender (being female) and receptiveness to complementary medicine. A negative association with receptiveness to conventional medicine approached statistical significance. Symptomatic avoidance of both wheat and dairy products was similarly predicted by being female, more receptive to complementary medicine and less receptive to conventional medicine. Symptomatic dairy avoidance was significantly predicted by age (negatively) and worry about illness. Follow-up Pearson point-biserial correlations revealed no significant associations between illness worry and a particular symptom or symptoms.

Table 4 shows the frequencies of past diagnoses reported by members of the three groups of avoiders relative to the rest of the (non-coeliac) sample. For each of the avoidance categories, membership was associated with increased reporting of a past diagnosis of food intolerance or allergy; for the SDA group, it was also associated with more reports of IBS and of chronic fatigue syndrome and for the SWDA group, with more reports of IBS and of depression.

One-way ANOVA were conducted to compare the ratings by the three avoidance groups of possible sources of influence on the decision to avoid. The sources of influence were defined as Medical (mean of Doctor and Other Specialist ratings), Complementary Medicine (mean of Naturopath and Other Complementary Medicine ratings), Family and Friends, and Media (mean of Internet and Media ratings). For the SWDA group, who completed influence ratings for both wheat avoidance and dairy avoidance, scores were further calculated as the mean of those ratings. Results showed a significant group difference for Complementary Medicine $(F(2,157)=6 \cdot 06$, $P=0.003)$; Tukey post hoc comparisons indicated that rated influence of complementary medicine sources 
Table 3 Multivariable logistic regression of predictors of symptomatic wheat avoidance, symptomatic dairy avoidance and symptomatic wheat-and-dairy avoidance; CSIRO Food and Health Survey, Australia, December 2010-February 2011

\begin{tabular}{|c|c|c|c|c|c|c|c|}
\hline \multirow[b]{2}{*}{ Predictor } & \multirow[b]{2}{*}{$\beta$} & \multirow[b]{2}{*}{ SE } & \multirow[b]{2}{*}{ Wald } & \multirow[b]{2}{*}{$P$} & \multirow[b]{2}{*}{ OR } & \multicolumn{2}{|c|}{$95 \% \mathrm{Cl}$ for $\mathrm{OR}$} \\
\hline & & & & & & Lower & Upper \\
\hline \multicolumn{8}{|l|}{ SWA $v$. rest of the sample } \\
\hline Receptiveness to complementary medicine & 0.49 & 0.16 & $9 \cdot 14$ & 0.002 & 1.64 & 1.19 & $2 \cdot 26$ \\
\hline Gender & -1.36 & 0.41 & $11 \cdot 17$ & 0.001 & 0.26 & 0.12 & 0.57 \\
\hline Receptiveness to conventional medicine & -0.29 & 0.15 & 3.73 & 0.053 & 0.75 & 0.56 & 1.00 \\
\hline Age & 0.01 & 0.01 & 0.94 & 0.333 & 1.01 & 0.99 & 1.03 \\
\hline Analytical thinking style & 0.22 & 0.21 & 1.09 & 0.297 & 1.24 & 0.83 & 1.88 \\
\hline Intuitive thinking style & 0.13 & 0.23 & 0.32 & 0.575 & 1.14 & 0.73 & 1.77 \\
\hline Neuroticism & -0.40 & 0.28 & $2 \cdot 11$ & 0.147 & 0.67 & 0.39 & 1.15 \\
\hline Illness worry & 0.09 & 0.20 & 0.19 & 0.662 & 1.09 & 0.73 & 1.63 \\
\hline \multicolumn{8}{|l|}{ SDA $v$. rest of the sample } \\
\hline Receptiveness to complementary medicine & 0.12 & 0.12 & $1 \cdot 13$ & 0.288 & $1 \cdot 13$ & 0.90 & 1.42 \\
\hline Gender & -0.09 & 0.24 & 0.16 & 0.692 & 0.91 & 0.57 & 1.45 \\
\hline Receptiveness to conventional medicine & -0.08 & 0.12 & 0.42 & 0.519 & 0.93 & 0.73 & 1.17 \\
\hline Age & -0.02 & 0.01 & 5.99 & 0.014 & 0.98 & 0.97 & 0.99 \\
\hline Analytical thinking style & 0.09 & 0.15 & 0.33 & 0.568 & 1.09 & 0.81 & 1.47 \\
\hline Intuitive thinking style & -0.13 & 0.16 & 0.64 & 0.423 & 0.88 & 0.65 & 1.20 \\
\hline Neuroticism & 0.05 & 0.18 & 0.07 & 0.799 & 1.05 & 0.74 & 1.49 \\
\hline Illness worry & 0.30 & 0.14 & 4.78 & 0.029 & 1.35 & 1.03 & 1.78 \\
\hline \multicolumn{8}{|l|}{ SWDA $v$. rest of the sample } \\
\hline Receptiveness to complementary medicine & 0.48 & 0.17 & $8 \cdot 21$ & 0.004 & 1.62 & $1 \cdot 16$ & $2 \cdot 25$ \\
\hline Gender & $-1 \cdot 30$ & 0.42 & 9.72 & 0.002 & 0.27 & 0.12 & 0.62 \\
\hline Receptiveness to conventional medicine & -0.62 & 0.14 & 18.52 & 0.000 & 0.54 & 0.41 & 0.71 \\
\hline Age & 0.01 & 0.01 & 0.89 & 0.345 & 1.01 & 0.99 & 1.03 \\
\hline Analytical thinking style & 0.40 & 0.22 & 3.30 & 0.069 & 1.49 & 0.97 & $2 \cdot 30$ \\
\hline Intuitive thinking style & 0.07 & 0.23 & 0.10 & 0.753 & 1.08 & 0.69 & 1.69 \\
\hline Neuroticism & -0.03 & 0.27 & 0.02 & 0.901 & 0.97 & 0.57 & 1.65 \\
\hline Illness worry & 0.30 & 0.21 & $2 \cdot 10$ & 0.147 & 1.35 & 0.90 & 2.01 \\
\hline
\end{tabular}

Model $X^{2}=90.42, P<0.001 ; n 1184$

Analyses involved a four-level classification of the sample as symptomatic wheat avoiders (SWA), symptomatic dairy avoiders (SDA), symptomatic wheat-anddairy avoiders (SWDA) and the rest of the sample with cases of coeliac disease excluded.

Table 4 Diagnosed conditions reported by symptomatic dairy avoiders (SDA), symptomatic wheat avoiders (SWA), symptomatic wheat-anddairy avoiders (SWDA) and the rest of the sample with cases of coeliac disease excluded; CSIRO Food and Health Survey, Australia, December 2010-February 2011

\begin{tabular}{|c|c|c|c|c|c|c|c|c|}
\hline \multirow[b]{2}{*}{ Reported diagnosed condition } & \multicolumn{2}{|c|}{ SDA } & \multicolumn{2}{|c|}{ SWA } & \multicolumn{2}{|c|}{ SWDA } & \multicolumn{2}{|c|}{ Rest of the sample } \\
\hline & $n$ & $\%$ & $n$ & $\%$ & $n$ & $\%$ & $n$ & $\%$ \\
\hline Heart disease and/or stroke & 5 & $6 \cdot 0$ & 5 & $11 \cdot 6$ & 5 & $12 \cdot 5$ & 114 & $11 \cdot 8$ \\
\hline Diabetes & 6 & $7 \cdot 1$ & 3 & $7 \cdot 0$ & 2 & $5 \cdot 0$ & 63 & 6.5 \\
\hline Obesity & 10 & $12 \cdot 2$ & 4 & $9 \cdot 8$ & 2 & $5 \cdot 1$ & 96 & $10 \cdot 0$ \\
\hline Bowel or colorectal cancer & 1 & 1.2 & 0 & 0.0 & 1 & 2.5 & 22 & $2 \cdot 3$ \\
\hline Irritable bowel syndrome & $13^{*}$ & $15 \cdot \overline{3}$ & 7 & $16 \cdot 7$ & $10^{\star \star}$ & $26 \cdot 3$ & 78 & $8 \cdot 1$ \\
\hline Inflammatory bowel disease & 4 & 4.7 & 2 & 4.7 & 3 & $7 \cdot 7$ & 24 & 2.5 \\
\hline Chronic fatigue syndrome & $7^{*}$ & $8 \cdot 2$ & 2 & 4.7 & 2 & $5 \cdot 1$ & 22 & $2 \cdot 3$ \\
\hline Asthma & 14 & $16 \cdot 5$ & 11 & $25 \cdot 6$ & 9 & 22.5 & 166 & $17 \cdot 4$ \\
\hline Depression & 23 & $27 \cdot 1$ & 12 & 27.9 & $15^{*}$ & 38.5 & 180 & $18 \cdot 8$ \\
\hline Food sensitivity or allergy & $33^{\star \star}$ & 37.5 & $16^{*}$ & $35 \cdot 6$ & $17^{\star \star}$ & 40.5 & 79 & 7.9 \\
\hline
\end{tabular}

${ }^{*} \chi^{2}$ significant at $<0.05$ level, ${ }^{* *} \chi^{2}$ significant at $<0.001$ level.

was significantly lower for the SDA group (mean $=1 \cdot 31$, $\mathrm{SD}=0.54)$ than for the SWDA group $($ mean $=1.74$, $\mathrm{SD}=0.76)$ but not the SWA group $($ mean $=1.46, \mathrm{SD}=0.63)$.

\section{Discussion}

The survey data indicate that a substantial number of the Australian adult population are avoiding dairy foods.
At $16.6 \%$ (population-weighted by age and gender), the prevalence of dairy avoidance reported is $55 \%$ greater than was observed for wheat ${ }^{(5)}$. The explanations provided for the behaviour include various idiosyncratic factors $(2 \cdot 8 \%)$, management of several (mainly cardiovascular) diagnosed medical conditions (1.2\%) and coeliac disease $(0.8 \%)$. However, of most interest here is the remaining $11.8 \%$ of the sample who attributed symptoms and negative reactions to the consumption of 
dairy foods. Almost a third $(3.6 \%)$ of this symptomatic group also reported avoiding wheat products for alleviation of symptoms. Together with wheat-only (3.5\%) and dairy-only $(8 \cdot 2 \%)$ avoiders, they put the combined prevalence of (ostensibly non-coeliac) symptomatic avoiders of either dietary factor at $15 \cdot 3 \%$.

\section{Symptoms, diagnoses and the potential for misattributions}

Milk and wheat both contain components that can trigger adverse serious physiological reactions. Principally, these components are a disaccharide (lactose) and protein (gluten), respectively, and the intolerances that they trigger are diagnosed clinically. However, the avoidance of dairy foods for symptom control, as found previously for wheat ${ }^{(5)}$, appeared to rely substantially on a nonmedically diagnosed connection between ingestion and symptoms. Reports of a diagnosed allergy or intolerance mandating the avoidance were similarly infrequent at $6.2 \%$ ( $0.7 \%$ of the sample). Avoiders of dairy foods cited gastrointestinal symptoms most commonly, although less consistently than was the case for wheat products. Fatigue was also cited less frequently and mucus build-up considerably more frequently for dairy than for wheat. Dairy products have often been blamed for increased oral and nasal mucus production, but these effects have not been substantiated by well-controlled studies ${ }^{(12,13)}$.

Although it would be unwise to attempt diagnoses on the basis of the present data, the symptoms claimed for dairy admit the possibility of undiagnosed dairy allergy or lactose intolerance. IBS-like symptoms may also reflect the involvement of poorly absorbed short-chain carbohydrates, other than lactose, which can induce gastrointestinal discomfort through osmotic effects or rapid fermentation by the colonic microbiota. These carbohydrates (FODMAPS: fermentable oligo-saccharides, disaccharides (lactose), monosaccharides (fructose) and polyols) ${ }^{(14,15)}$ may be present in dairy products or in foods consumed with dairy products, and effects may be independent of or additive with lactose ${ }^{(16)}$.

Reports of past diagnoses elicited independently of the question of food avoidance do not clarify the picture greatly. Symptomatic avoiders of dairy, wheat or both often reported having at some time been diagnosed with a food intolerance or allergy despite the dearth of such reports specific to the avoidance behaviour. Unsurprisingly, reports of a previous diagnosis of IBS were also common. Dairy avoiders were also more likely than the average to report a past diagnosis of chronic fatigue syndrome, although the numbers involved were still small. It has been proposed that a co-morbid triad of perceived food intolerance, IBS and fatigue might point to a common underlying cause ${ }^{(17)}$; however, a causal relationship between these conditions has not been ascertained. Causation is also unclear in the greater likelihood for dairy avoiders who also avoided wheat to report a past diagnosis of depression.

Whatever the causal agents and physiological factors that trigger the avoidance of dairy foods, the general lack of a formal diagnosis - reasons for which may range from negative test results to an absence of medical involvement - is a matter of serious public health concern. It has been proposed that health-care professionals may recommend avoidance of dairy foods as a viable treatment option when they feel that they have little else to offer patients ${ }^{(18)}$. Self-diagnosed links between a dietary component and symptoms may derive from adverse publicity either directly or as a manifestation of categorical thinking about some foods as being intrinsically bad ${ }^{(19)}$. Although consumers may find justification for a dietary strategy in an apparent reduction in symptoms, confidence may be misplaced ${ }^{(20)}$. Non-specific gastrointestinal symptoms often attributed to intolerance have been found to be susceptible to the placebo effect associated with dietary manipulations ${ }^{(21-24)}$. In short, the potential for misattribution of an underlying symptomatic state is clearly considerable.

\section{Two populations of avoiders?}

The similarities in symptoms and frequent co-occurrence of dairy avoidance and wheat avoidance prompt the question whether they are fundamentally two expressions of a single phenomenon. However, the different predictive profiles broadly suggest two avoidant populations: the one avoiding wheat and possibly also dairy foods and another avoiding dairy but not wheat foods. They also depict the two types of avoidance as substantially different psychosocial phenomena, with dairy avoidance tending to be associated with an internal focus on the possibility of illness rather than the external focus on treatment strategies evident in wheat avoidance.

Worry about illness may represent a position on a continuum of health anxiety of which hypochondriasis is an extreme and rare form ${ }^{(25-28)}$. Concerns about the possibility of illness may be also be reactive, being provoked by perceived unusual changes in bodily functioning, exposure to negative information about health that is personally significant, or (perhaps less plausibly here, given the null finding for neuroticism) bodily sensations that are catastrophically interpreted ${ }^{(29)}$. What cannot be determined, therefore, is to what degree dairy avoidance is the behaviour of a more symptom-vigilant population ${ }^{(30)}$ or associated with more severe or distressing symptoms ${ }^{(31)}$. This is an important distinction and a question for further research.

\section{Strengths, limitations and public bealth implications}

As discussed previously ${ }^{(5)}$, the strengths of these survey data lie in the use of probability sampling from the 
national adult population and the potential contributions of response anonymity and structure of the omnibus survey to limiting of response biases. Conversely, weaknesses include the inability to substantiate participants' reports, to examine possible differences in dairy avoidance by race or ethnicity, or to quantify any possible sampling bias associated with interest in food or health. If these data are representative, they document symptom-related avoidance of wheat or dairy products by one in seven Australian adults of voting age. Not included in this estimate are those further adults (estimated at 5.8\%) with symptomunrelated explanations for their avoidance, other household members exposed to these dietary strategies and children. Even if the survey figures should overestimate prevalence, they testify to a significant public health phenomenon in the self-prescribed avoidance of dietary factors.

The self-prescribed nature of symptom-related dairy (and wheat) avoidance inevitably raises concerns about the rigour and accuracy of the diagnostic process and the risk of serious health conditions going undetected. A more serious issue arises if the elimination of a dietary factor is naively assumed to be intrinsically health-enhancing without compensatory replacement of sources of the missing nutrients. Irrespective of whether or not individuals are correct in identifying dairy products as the cause of their symptoms, we then have the paradoxical prospect of a health-motivated reduction in dairy consumption increasing risk of a condition like osteoporosis. Moreover, the volitional nature of a self-prescribed intervention lends it an added psychological potency. With a sense of autonomy come enhanced ownership of, adherence to and consequently outcomes of a health intervention ${ }^{(32,33)}$. In the present case, this can be expected to increase also the likelihood of any adverse consequences.

\section{Future directions}

Clearly, these survey findings warrant follow-up investigation to determine both the decision processes leading symptomatic individuals to avoid dairy consumption and the impact on resultant nutritional status. Given the potential numbers of Australians involved, it is important to establish whether increased level of worry about illness is antecedent to or a response to symptoms. The latter suggests that symptom severity may be a factor. Whether the attribution of symptoms to dairy is accurate is a matter for clinical investigation. However, the process is further complicated in the case of people who are also avoiding wheat products. Further questions for investigation include what other self-prescribed dietary changes consumers may be practising, whether in response to symptoms or the perception that such behaviour is intrinsically health-promoting. It is also noteworthy that few avoiders of dairy invoked lactose in explaining their behaviour. This cautions against the conclusion that the behaviour observed here substantially explains the market for lactose-free products; a similar point was made in respect of wheat avoidance and the gluten-free market ${ }^{(5)}$. Nor should the behaviour be interpreted as evidence of self-diagnosed food intolerances, which have been the topic of much discussion in recent decades ${ }^{(34-36)}$. A perceived intolerance is a particular attribution of a symptomatic state and may thus represent the response of a subset only of those who avoid a dietary factor because of symptoms they associate with its consumption. It is the avoidance that especially demands systematic investigation.

\section{Conclusions}

In addition to the many adult Australians avoiding consumption of wheat or both wheat and dairy products, a similar further number reports avoiding dairy products, similarly mostly without a formal diagnosis, and citing largely similar, primarily gastrointestinal, symptoms. The accuracy of self-diagnoses, the actual sources of symptoms and the physiological mechanisms remain to be established; in addition, the tendency for dairy avoidance to be associated with more worry about illness identifies both symptom severity and psychological responses to symptoms attributed to dairy as targets for further investigation. Most significantly, though, the findings are further evidence of a widespread tendency for people to seek to exercise control over their health by eliminating dietary factors considered suspect without medical evidence or oversight. Avoiding foods to alleviate adverse symptoms should be weighed against the consequences of eliminating dietary factors and their related nutrient profiles. In the case of dairy foods, those consequences could be significant for individuals and, given the apparent scale of the avoidance behaviour, for society in the long term.

\section{Acknowledgements}

Financial support: This study was part of a larger project conducted with financial assistance from the Grains Research and Development Corporation. The funding body had no involvement in the design of the study, collection, analysis or interpretation of the data, writing of the report, or the decision to submit the article for publication. Conflict of interest: The authors have no conflict of interest to disclose. Authorship: P.M. and S.G. designed and conducted the study. B.Y. conducted data analysis. B.Y. and P.M. drafted the manuscript, and all authors were involved in critical revision of the manuscript. All authors had full access to all of the data and can take responsibility for the integrity of the data and the accuracy of the data analysis. Ethics of buman subject participation: The study was approved by the CSIRO Human Research Ethics Committee (LR17/2010). 


\section{References}

1. Weaver CM (2009) Should dairy be recommended as part of a healthy vegetarian diet? Point. Am J Clin Nutr 89, issue 5, 1634S-1637S.

2. Carroccio A, Montalto G, Cavera G et al. (1998) Lactose intolerance and self-reported milk intolerance: relationship with lactose maldigestion and nutrient intake. $\mathrm{J} \mathrm{Am} \mathrm{Coll}$ Nutr 17, 631-636.

3. Appleby P, Roddam A, Allen N et al. (2007) Comparative fracture risk in vegetarians and non-vegetarians in EPICOxford. Eur J Clin Nutr 61, 1400-1406.

4. Australian Bureau of Statistics (2011-12) Australian Health Survey: Nutrition First Results - Foods and Nutrients, 2011-12. National Nutrition and Physical Activity Survey (NNPAS). http://www.abs.gov.au/ausstats/abs@.nsf/mf/4364.0.55.007 (accessed November 2015).

5. Golley S, Corsini N, Topping D et al. (2015) Motivations for avoiding wheat consumption in Australia: results from a population survey. Public Health Nutr 18, 490-499.

6. Goldberg L, Johnson JA, Eber HW et al. (2006) The international personality item pool and the future of publicdoman personality measures. J Res Pers 40, 84-96.

7. Epstein S, Pacini R, Denes-Raj V et al. (1996) Individual differences in intuitive-experiential and analytical-rational thinking styles. J Pers Soc Psychol 71, 390-405.

8. Cacioppo J \& Petty RE (1982) The need for cognition. J Pers Soc Psychol 42, 116-131.

9. Pilowsky I (1967) Dimensions of hypochondriasis. $\mathrm{Br} J$ Psychiatry 113, 89-93.

10. Little RJA (1988) A test of missing completely at random for multivariate data with missing values. J Am Stat Assoc 83, 1198-1202.

11. Siegel S (1956) Nonparametric Statistics for the Behavioural Sciences. New York: McGraw-Hill Book Company, Inc.

12. Wuthrich B, Schmid A, Walther B et al. (2005) Milk consumption does not lead to mucus production or occurrence of asthma. J Am Coll Nutr 24, Suppl. 6, 547S-555S.

13. Pinnock C, Graham NM, Mylvaganam A et al. (1990) Relationship between milk intake and mucus production in adult volunteers challenged with rhinovirus-2. Am Rev Respir Dis 141, 352-356.

14. Gibson P \& Shepherd SJ (2005) Personal view: food for thought - western lifestyle and susceptibility to Crohn's disease. The FODMAP hypothesis. Aliment Pharmacol Ther 21, 1399-1409.

15. Gibson P \& Shepherd SJ (2010) Evidence-based dietary management of functional gastrointestinal symptoms: the FODMAP approach. J Gastroenterol Hepatol 25, 252-258.

16. Shepherd S, Parker FC, Muir JG et al. (2008) Dietary triggers of abdominal symptoms in patients with irritable bowel syndrome: randomized placebo-controlled evidence. Clin Gastroenterol Hepatol 6, 765-771.

17. Simren M, Abrahamsson H, Svedlund J et al. (2001) Quality of life in patients with irritable bowel syndrome seen in referral centers versus primary care: the impact of gender and predominant bowel pattern. Scand J Gastroenterol 36, 545-552.

18. Heaney R, Keith JN \& Duyff RL (2011) Unintended consequences of dairy avoidance. http://www.nationaldairy council.org/SiteCollectionDocuments/health_wellness/lactose_ intolerance/Unintended\%20Consequences\%200f\%20Dairy\%20 Avoidance\%20-\%202011.pdf (accessed November 2015).
19. Rozin P, Ashmore M \& Markwith M (1996) Lay American conceptions of nutrition: dose insensitivity, categorical thinking, contagion, and the monotonic mind. Health Psychol 15, 438-447.

20. Suarez F \& Levitt MD (1996) Abdominal symptoms and lactose: the discrepancy between patients' claims and the results of blinded trials. Am J Clin Nutr 64, 251-252.

21. Suarez F, Savaiano DA \& Levitt MD (1995) A comparison of symptoms after the consumption of milk or lactose-hydrolyzed milk by people with self-reported severe lactose intolerance. $N$ Engl J Med 333, 1-4.

22. Rorick M \& Scrimshaw NS (1979) Comparative tolerance of elderly from differing ethnic backgrounds to lactose-containing and lactose-free dairy drinks: a double-blind study. J Gerontol 34, 191-196.

23. Vesa T, Korpela RA \& Sahi T (1996) Tolerance to small amounts of lactose in lactose maldigesters. Am J Clin Nutr 64, 197-201.

24. Johnson A, Semenya JG, Buchowski MS et al. (1993) Adaptation of lactose maldigesters to continued milk intakes. Am J Clin Nutr 58, 879-881.

25. Faravelli C, Salvatori S, Galassi F et al. (1997) Epidemiology of somatoform disorders: a community survey in Florence. Soc Psychiatry Psychiat Epidemiol 32, 24-29.

26. Noyes R, Happel RL \& Yagla SJ (1999) Correlates of hypochondriasis in a non-clinical population. Psychosomatics $\mathbf{4 0}$, 461-469.

27. Lieb R, Pfister H, Mastaler M et al. (2000) Somatoform syndromes and disorders in a representative population sample of adolescents and young adults: prevalence, comorbidity, and impairments. Acta Psychiatr Scand 101, 194-208.

28. Looper K \& Kirmayer LJ (2001) Hypochondriacal concerns in a community population. Psychol Med 31, $577-584$

29. Sayar K, Kirmayer LJ \& Taillefer SS (2003) Predictors of somatic symptoms in depressive disorder. Gen Hosp Psychiatry 25, 108-114.

30. Yang J, Deng Y, Chu $\mathrm{H}$ et al. (2013) Prevalence and presentation of lactose intolerance and effects on dairy product intake in healthy subjects and patients with irritable bowel syndrome. Clin Gastroenterol Hepatol 11, 262-268.

31. McBean L \& Miller GD (1998) Allaying fears and fallacies about lactose intolerance. J Am Diet Assoc 98, 671-676.

32. Deci E \& Ryan RM (2008) Self-Determination Theory: a macrotheory of human motivation, development, and health. Can Psychol 49, 182-185.

33. Ryan R, Patrick H, Deci EL et al. (2008) Facilitating health behaviour change and its maintenance: interventions based on self-determination theory. Eur Health Psychol 10, 2-5.

34. Rona R, Keil T, Summers C et al. (2007) The prevalence of food allergy: a meta-analysis. J Allergy Clin Immunol 120, 638-646.

35. Pearson D, Rix KJB \& Bentley SJ (1983) Food allergy: how much in the mind? A clinical and psychiatric study of suspected food hypersensitivity. Lancet $\mathbf{1}$, 1259-1261.

36. Stein K (2009) Are food allergies on the rise, or is it misdiagnosis? J Am Diet Assoc 109, 1832, 1834, 1836-1837. 